\title{
Quantification of Lower Urinary Tract Function/Dysfunction
}

\author{
R. van Mastrigt \\ Department of Urology, Erasmus University, Rotterdam, The Netherlands
}

Key words: urinary system, treatment modalities, parameters, voiding function, contractility, urethral resistance, obstruction

\section{INTRODUCTION}

The purpose of urodynamic investigation is to derive information on the function of the urinary system. Decisions on the applicability and effect of treatment modalities are based on this information that should therefore be objective and quantitative. Moreover the information should be selective so that functionally and conceptually different parts of the (urinary) system are represented by separate parameters. The urinary system comprises three conceptually different subsystems, the detrusor muscle, the urethra, and the (neurogenic) system that controls contraction and relaxation of both these anatomical structures. Three important aspects of the function of these subsystems are the contractility of the bladder during voiding, its stability during filling, and the resistance to flow of the urethra during voiding.

\section{MATERIALS AND METHODS}

Computer software has been developed that enables the analysis of the detrusor pressure and urinary flow rate signals measured during filling and voiding cystometry in terms of contractility, (in)stability, and urethral resistance (CLIM) [van Mastrigt, 1984]. The contractility of the bladder can be characterized in terms of a relation between the pressure that it generates and the shortening velocity of the bladder wall. This relation reflects the basic force-velocity relationship of smooth muscle. In the program it is quantified by the parameter wmax that combines both these aspects of contractility in the form of an approximated power per bladder wall surface area [Griffiths et al., 1986]. The degree of instability of the detrusor during filling can be quantified by integration of this approximated power in each unstable contraction

Received for publication January 10, 1992; accepted January 24, 1992.

Address reprint requests to R. van Mastrigt, Department of Urology, Room EE1630, Erasmus University, 3000 DR Rotterdam, The Netherlands.

This paper was presented at the Microsymposia on Lower Urinary Tract Dysfunction, Erasmus University, Rotterdam, December 7, 1991. 
[Klijn et al., 1990]. The urethral resistance is defined by the relation between the pressure drop over the urethra and the flow rate and is quantified by the parameter URA that estimates the intersection of the pressure-flow rate curve on the pressure axis [Griffiths et al., 1989].

\section{RESULTS}

The computer program CLIM was applied in a number of clinical studies to derive the contractility parameter wmax and the urethral resistance parameter URA with the following results.

In a pilot study on ten patients $(H$. Jansen and $R$. van Mastrigt, unpublished data, 1992) URA and wmax were correlated with the diameter of the urethra at a number of locations along its length, as determined by transrectal ultrasonography. It was concluded that there was a significant (negative) correlation between URA and the urethral diameter, and there was no significant correlation of this diameter with the contractility parameter wmax, indicating that wmax is a selective parameter in the sense that it is not biased by urethral resistance.

In a study on a mixed group of 241 patients, from which 66 were obstructed and 175 were unobstructed according to the parameter URA, it was found that there was no significant difference in the maximum contraction velocity of both groups of patients, but there was a difference in the isometric detrusor pressure [van Mastrigt, 1990]. As wmax is a combination of these two aspects of contractility, it was different for both groups of patients, i.e., the obstructed patients had a significantly higher wmax, secondary to the obstruction. In the group of 175 unobstructed patients there were 38 patients with more than $50 \mathrm{ml}$ of residual urine after voiding. These patients had a significantly smaller wmax value than the 137 patients without residual urine, showing that in these unobstructed patients the residual urine was caused by a poor contractility of the bladder.

In a group of 29 patients who underwent trans urethral resection of the prostate on the basis of conventional clinical diagnostics the contractility of the bladder and the urethral resistance were evaluated before and after surgery [Rollema and van Mastrigt, 1992]. It was found that upon objective evaluation ten patients in this group were not obstructed, but the surgery was nevertheless performed on clinical grounds. In the 19 obstructed patients the urethral resistance parameter URA was significantly decreased after the operation, and the maximum flow rate was accordingly increased. In the ten unobstructed patients these changes did not occur. In both groups there was no significant change in the value of the parameter wmax due to the operation, confirming that dramatic changes in urethral resistance do not influence the contractility parameter wmax. It was furthermore shown that using this parameter it can pre-operatively be predicted which patients will postoperatively still void with a significant amount of residual urine [van Mastrigt and Rollema, 1988].

The effect of the anticholinergic drugs oxyphenonium bromide and oxybutynin $\mathrm{HCl}$ on detrusor contractility and vesicoureteral reflux was studied in a group of 12 children [Scholtmeijer and van Mastrigt, 1991]. It was found that there was no significant reduction in the reflux score of children treated with oxyphenonium bromide, nor was there such a reduction in a control group of 12 children only treated with antibiotics. In the children treated with oxybutynin $\mathrm{HCl}$, however, a significant reduction in the reflux score was found. This reduction was paralleled by a significant 
decrease in bladder contractility as quantified by wmax in these children. Such a decrease was not found in the control group and in the children treated with oxyphenoniumbromide.

In a trial of a selective alpha-1 adrenoreceptor-blocking agent in a group of benign prostatic hypertrophy patients, it was shown that this drug (Doxazosin) significantly reduced URA, but did not effect the contractility parameter wmax [Rollema et al., 1991].

In a small test series of 15 patients, the described instability parameter was calculated from the recordings of both standing and supine filling cystometry procedures. A significant difference between these values could be shown [Klìjn et al., 19901.

\section{CONCLUSION}

In order to accurately and objectively evaluate the function and dysfunction of the urinary tract, so that the effect of different treatment modalities on different patients can be compared, it is necessary to quantitatively and selectively characterize the functionally different parts of the system. The described parameters and software form a means to this end.

\section{REFERENCES}

Griffiths DJ, Constantinou CE, van Mastrigt R (1986): Urinary bladder function and its control in normal females. Am J Physiol 251-2:R225-R230.

Griffiths D, van Mastrigt R, Bosch R (1989): Quantification of urethral resistance and bladder function during voiding, with special reference to the effects of prostate size reduction on urethral obstruction due to BPH. Neurourol Urodyn 8:17-27.

Klijn AJ, van Mastrigt R, Bosch JLHR (1990): An energy-based parameter for detrusor instability, Neurourol Urodyn 9:385-387.

Rollema HJ, Rosier P, Janknegt RA, van Mastrigt R (1991): Efficacy of alpha-blocker (Doxazosin) in BPH appraised by pressure-flow (CLIM) analysis. Neurourol Urodyn 10:295-296.

Rollema JH, van Mastrigt R (1992): Improved indication and follow-up in transurethral resection of the prostate (TURP) using the computer program CLIM: A prospective study. J Urol 147 (in press).

Scholtmeijer RJ, van Mastrigt R (1991): The effect of oxyphenonium bromide and oxybutynin $\mathrm{HCl}$ on detrusor contractility and reflex in children with vesicoureteral reflux and detrusor instability. $\mathrm{J}$ Urol 146:660-662.

van Mastrigt R (1984): A computer program for on-line measurement, storage, analysis and retrieval of urodynamic data. Comp Prog Biomed 18:109-117.

van Mastrigt R, Rollema HJ (1988): Urethral resistance and urinary bladder contractility before and after transurethral resection as determined by the computer program CLIM. Neurourol Urodyn 7:226-228.

van Mastrigt R (1990): Estimation of the maximum contraction velocity of the urinary bladder from pressure and flow throughout micturition. Urol Res 18:149-154. 